\title{
Algebraic Quantization on a Group and Nonabelian Constraints ${ }^{\star}$
}

\author{
Victor Aldaya $^{1 \star}$, Jose Navarro-Salas ${ }^{2,3}$ and Alfonso Ramirez ${ }^{2}$ \\ 1 The Blackett Laboratory, Imperial College, London SW7 2BZ, England \\ 2 Departamento de Fisica Teorica, Facultad de Fisicas, Universidad de Valencia, Burjasot E-46100 \\ Valencia, Spain \\ ${ }^{3}$ Centro Mixto Universidad de Valencia, C.S.I.C., Burjasot E-46100 Valencia, Spain
}

\begin{abstract}
A generalization of a previous group manifold quantization formalism is proposed. In the new version the differential structure is circumvented, so that discrete transformations in the group are allowed, and a nonabelian group replaces the ordinary (central) $U(1)$ subgroup of the Heisenberg-Weyl-like quantum group. As an example of the former we obtain the wave functions associated with the system of two identical particles, and the latter modification is used to account for the Virasoro constraints in string theory.
\end{abstract}

\section{Introduction}

The use of group theoretical formulations has increased, since the pioneer work by Ne'eman and Regge [1], making easier and more natural the incorporation of infinite dimensional symmetry groups to dynamical systems and generalizing the basic quantum structures. In general terms the nonlinear sigma model on group manifolds $[2,3]$ and string theory on group manifolds [4-6] are examples of modern formulations where the Lie structure of a certain group plays an important role (see also refs. [7-11] and references therein). It constitutes a step towards the algebrization of physical theories, an evolution analogous with that previously suffered by Mathematics itself.

In this paper we present a generalization of a group approach to quantization $[12-14]$ in which a Lie group $\widetilde{G}$ and its canonical structures were the only starting points (see also ref. [13] and references therein). The structure of the group itself

\footnotetext{
* Research partially supported by the Conselleria de Cultura de la Generalitat Valenciana, the Plan de Formacion del Personal Investigador, and the Comision Asesora de Investigacion Cientifica y Tecnica (CAICYT)

$\star \star$ On leave of absence from the IFIC, Centro Mixto Universidad de Valencia-C.S.I.C. and the Departamento de Fisica Teorica de la Universidad de Valencia
} 
was that of a principal bundle with structure group $U(1)$ (or $R$ for the classical limit). Although central extensions were mainly employed, some nontrivial bundles were considered as well $[15,10]$.

The idea of a formulation of dynamics on a group manifold has support in the strong parallelism between the configuration space of certain physical elementary systems and the corresponding symmetry groups. To be more precise the comparison is best established between a generalized configuration space including the evolution of the wave function (or the Hamilton principal function) and the group of transformations leaving the Lagrangian strictly invariant. For concreteness, let us consider the free spinless particle in three dimensions. The lagrangian $(1 / 2) m \dot{x}^{2}$, defined on $M \equiv R^{3} x R^{3} x R$, is not strictly invariant under the action of the Galilei group $\mathbb{G}$. This fact is not due to the structure of the space $M$ considered but a consequence of the non-trivial cohomology of $\mathbb{G}$ with values in the co-adjoint representation [16]. The symplectic cohomology group $H^{1}\left(\mathbb{G}, \mathscr{G}^{*}\right)$ (where $\mathscr{G}^{*}$ is the dual to the Lie algebra $\mathscr{G}$ of $\mathbb{G}$ ) or equivalently $H^{2}(\mathbb{G}, U(1))$ [17] (see also ref. [12]) is an obstruction to the existence of strictly invariant lagrangians. The minimal modification to be made in order to break down that obstruction is either to extend the manifold $M$ by $S^{1}$ (respectively $R$ ) and the group $\mathbb{G}$ by $U(1)$ (respectively the additive group $R$ ) [14]. The simplest non-trivial Lagrangian which is strictly invariant under the $U(1)$ central extension $\tilde{\mathbb{G}}_{(m)}$ (respectively $R$ central extension $\left.\overline{\mathbb{G}}_{(m)}\right)$ is that leading to the Schrödinger (respectively Hamilton-Jacobi) equation for the free particle. ${ }^{1}$

The parallel between the extended configuration space $\tilde{M}=M x S^{1}$ and the group manifold of $\widetilde{\mathbb{G}}_{(m)}$ is that the former is the manifold of the quotient group $\widetilde{\widetilde{G}}_{(m)} / S O(3)$. For the solution manifold we have $\tilde{\mathbb{G}}_{(m)} /\{S O(3) \times$ time translations $\}$, i.e., the quotient of $\widetilde{\mathbb{G}}_{(m)}$ by the subgroup which plays no role in the group extension process. It is in fact a quantum manifold in the sense of refs. [16] and [27]. Working on the bigger manifold $\widetilde{\mathbb{G}}_{(m)}$ requires, however, the incorporation of certain restrictions on the wave function $\Psi$ (Hamilton principal function $S$ in the case of $\left.\widetilde{\mathbb{G}}_{(m)}\right)$ associated with the non-symplectic parameters of $S O(3)$, time translations and $U(1)(R)$ subgroups. In particular, the restriction associated with $U(1)$, denominated $U(1)$-equivariant condition, is written as $\Psi(\zeta * \tilde{g})=\zeta \Psi(\tilde{g}), \zeta \in U(1)$, $\tilde{g} \in \tilde{\mathbb{G}}_{(m)}$ or $i \zeta \partial \Psi / \partial \zeta=i \Psi$ in the infinitesimal form $\left(S(\phi * \bar{g})=\phi+S(\bar{g}), \phi \in R, \bar{g} \in \overline{\mathbb{G}}_{(m)}\right.$ or $\partial S / \partial \phi=1)$. The remainder appears as a part of a generalized polarization $[12,14]$ (see refs. [16] and [27] for the standard definition of polarization).

The above features corresponding to the free particle can be extended to numerous finite-dimensional systems [14] as well as infinite-dimensional ones as a result of a perturbative method of computing group laws [10] which, in principle,

\footnotetext{
${ }^{1}$ It must be remembered that the non-extended Galilei group $\mathbb{G}$ does not leave invariant the wave function of the free particle. Under a boost parametrized by $\underline{V}, \Psi(r, t)$ behaves as $\Psi^{\prime}\left(r^{\prime}, t^{\prime}\right)=$ $\exp (i m / \hbar)\left\{1 / 2 \underline{V}^{2} t+\underline{V} \cdot \underline{R} \underline{\underline{r}}\right\} \Psi(\underline{r}, t) \equiv \exp (i m / \hbar) \xi(g ; \underline{r}, t)$, where $m$ is the mass. The cocycles of $H^{2}(G, U(1))$ have the form $\xi\left(g ; g^{\prime}\right)$, now $g, g^{\prime} \in \mathbb{G}$, except for an arbitrary coboundary, i.e., $\xi_{\mathrm{cob}}\left(g_{1}, g_{2}\right) \equiv \eta\left(g_{1} * g_{2}\right)-$ $\eta\left(g_{1}\right)-\eta\left(g_{2}\right)$, where $\eta$ is a real function on $\mathbb{G}$. For the Hamiltonian principal "wave" function we have equivalent expressions: $S^{\prime}\left(\underline{r}^{\prime}, t^{\prime}\right)=m \xi(g ; \underline{r}, t)+S(\underline{r}, t)$. Nevertheless the $\hbar$ constant can now be discarded as the function $S$ already incorporates the dimension of an action
} 
enable us to apply the quantization formalism to any Lie group $\widetilde{G}$. Thus, a vast number of dynamical systems could arise as associated with classified groups. In each case the quantization procedure seems to be quite clear although calculations are definitely non-trivial.

The generalization that we propose here is twofold. On the one hand we want the formalism to incorporate discrete transformations on the same footing as continuous ones. This means that discrete transformations can appear, not only connecting two separate sectors of the theory as in certain gauge field theories (see ref. [18] and references therein), but also playing the role of co-ordinate-momentum pairs as in the case of quantum strings on a torus [19]. On the other hand the structure group $U(1)$ (or $R$ ) of the quantum group $\tilde{G}$ viewed as a principal bundle must be, in general, replaced by a group $T$ which need not be abelian and which contains some $U(1)$ (or $R$ ) subgroup. Our intention in so doing is, in particular, to generalize the $U(1)$-function condition on the wave functions, $i \zeta \partial \Psi / \partial \zeta=i \Psi$, in order to accommodate non-abelian constraints in a natural and consistent manner, i.e., compatible with the polarization conditions. Non-abelian constraints have to be imposed when quantizing nonabelian gauge theories [20] as well as string theories [21,22] (a partial study of constraints was already made [13] in the framework of the previous formalism [12]). As a by-product the quantization 1 -form [12], which is a connection 1-form on the principal bundle [23] $\widetilde{G}$, acquires new (vertical) components (those on the structural group $T$ of $\widetilde{G}$ ) transforming as ghosts under $T$. We will not be concerned here with ghost nor any other element of the BRST formalism [24,25] but we refer to ref. [26], where the transformation properties of the vertical components of the connection 1-form are analysed. Another particularity of "non-abelian" quantization is that the $\hbar$ constant is included in a set of constants characterizing the irreducible representations of $T$. $\hbar$ was associated with the $U(1)$ group law, usually joined to the cocycle $\xi\left(g^{\prime}, g\right)$ to compensate its dimensions (see footnote 1).

Two quite different examples have been chosen to illustrate the generalized quantization formalism. We consider one in which a discrete transformation is an essential ingredient of the theory and another one where the essential point is the raising of constraints as a consequence of enlarging the structural group. The first one is the quantization of two ( $n$ in general) free identical particles, the discrete transformation being that of identifying both. The second one, not so simple, has the group Virasoro (S) $\{$ Affine $\Omega S U(2)\}[11]$ as $\tilde{G}$ and the dynamics is closely related to that of strings on the $S U(2)$ group.

The organization of the paper is as follows. In Sect. II we motivate the general theory with the help of the free particle example and present the essential structure of the group manifold quantization approach. After commenting on the kind of modifications required by the presence of discrete transformations in $\tilde{G}$ or nonabelian ones in the structural group, we generalize in Sect. III the previous formalism following a parallel exposition. Section IV is devoted to the example of the $n$ identical particles system whose associated quantum group $\widetilde{G}$ contains discrete transformations. Finally, in Sect. V we apply the generalized formalism to a particular fibration on the group Virasoro (S) Affine $\Omega S U(2)\}$ the fibre (structural group) of which contains the Virasoro subgroup. 


\section{Quantization Formalism on a Connected Lie Group: The Free Galilean Particle}

The group approach to (Geometric) Quantization [12-14] generalizes the mathematical structure underlying the symmetry transformations associated with quantities appearing in the basic Poisson brackets

$$
\left\{m V_{i}, K^{j}\right\}=\delta_{i}^{j}, \quad i, j=1, \ldots, n .
$$

These quantities are conjugate co-ordinates and momenta of the symplectic manifold of solutions of a mechanical problem. The commutation relations (2.1) are none other than the only nontrivial commutators of the Heisenberg-Weyl group $\tilde{H} W_{(m)}$, an $U(1)$ central extension of the additive group of the symplectic vector space of dimension $2 n$ [16]. Its group law is written

$$
\begin{array}{rlrl}
A^{\prime \prime i} & =A^{\prime i}+A^{i}, & & A \in R^{n}, \\
V^{\prime \prime j} & =V^{\prime j}+V^{j}, & & V \in R^{n}, \\
\zeta^{\prime \prime} & =\zeta^{\prime} \zeta \exp \left[(i / 2 \hbar) m \omega\left(g^{\prime}, g\right)\right], & \zeta \in U(1),
\end{array}
$$

where $g$ represents the element $\left(A^{i}, V^{j}\right), \omega$ is the canonical symplectic form of the symplectic vector space, $m$ is a positive real number, the mass, and $\hbar$ is the Planck constant introduced to make the exponent dimensionless. The relations (2.1) are also the commutation relations among (right-) invariant vector fields of $\widetilde{H} W_{(m)}$ once the identifications $\tilde{X}_{\left(A^{i}\right)}^{R} \rightarrow P_{i} \equiv m V_{i}, 1 / m \tilde{X}_{\left(V^{j}\right)}^{R} \rightarrow K_{j}, \tilde{X}_{(\zeta)}^{R} \rightarrow 1$ have been made. An irreducible representation of the Poisson algebra $\left\{P_{i}, K^{j}\right\}=\delta_{i}^{j},\left\{P_{i}, P_{j}\right\}=0=$ $\left\{K^{i}, K^{j}\right\}$ which assigns $i \hbar$ to the function 1 (i.e. a quantization) is achieved by letting $\tilde{X}_{\left(A^{1}\right)}^{R}, \tilde{X}_{\left(V^{j}\right)}^{R}$ act, as ordinary derivations, on functions $\Psi$ on $\tilde{H} W_{(m)}$ which are $U(1)$-equivariant (i.e. $\tilde{X}_{(\zeta)} \cdot \Psi=i \Psi$ ) and which have null Lie derivative along the direction of the left-invariant vector field $\widetilde{X}_{\left(A^{2}\right)}^{L}$ which, in the costomary language of the geometric quantization, generate what is called a polarization $[16,27,28]$.

A more complete example of what later on in this section will be called a dynamical (or "quantum") group is provided by enlarging the group law (2.2) by some rotation and evolution parameters. The following group law, which defines a central extension $\widetilde{\mathbb{G}}_{(m)}$ of the Galilei group $\mathbb{G}$ (see references in [12]) constitutes a simple example:

$$
\begin{aligned}
& B^{\prime \prime}=B^{\prime}+B, \\
& \underline{A}^{\prime \prime}=\underline{A}^{\prime}+\underline{R}^{\prime} \underline{A}+\underline{V}^{\prime} B, \\
& \underline{V}^{\prime \prime}=\underline{V}^{\prime}+\underline{R}^{\prime} \underline{V}, \\
& \underline{R}^{\prime \prime}=\underline{R^{\prime}} \underline{R}, \\
& \zeta^{\prime \prime}=\zeta^{\prime} \zeta \exp i m /(2 \hbar)\left[\underline{R^{\prime}} \underline{V} \cdot \underline{A^{\prime}}-\underline{R^{\prime}} \underline{A} \cdot \underline{V}^{\prime}+\underline{V}^{\prime} \cdot \underline{R^{\prime}} \underline{A} B\right] .
\end{aligned}
$$

Here we have made use of one of the possible cocycles or local exponents $\xi\left(g^{\prime}, g\right)$ of the cohomology group $H^{2}(\mathbb{G}, U(1))$ of the Galilei group $\mathbb{G}$; the classes in $H^{2}(\mathbb{G}, U(1))$ are parametrized by the (positive) real number $m$. Expression (2.3) will correspond to the spinless free particle of mass $m$ [12].

The important feature of a group law like (2.3) is its principal bundle structure with fibre $U(1)$ and base $\widetilde{\mathbb{G}}_{(m)} / U(1)$ as well as the fact that the fibration is canonical. The fibration distinguishes one component of the left-invariant one-form $\theta^{L}$ on 
$\tilde{\mathbb{G}}_{(m)}$ : the vertical component which in local co-ordinates is just the component $\theta^{L(\zeta)}$ dual to the fundamental vector field $i \zeta \partial / \partial \zeta \equiv \Xi$. This ordinary ( $R$-valued) one-form, which in this case is given by

$$
\Theta=(m / 2)[\underline{V} \cdot d \underline{A}-\underline{A} \cdot d \underline{V}]-(1 / 2 m) V^{2} d B+\hbar d \zeta /(i \zeta),
$$

is a pre-contact one-form on the manifold $\tilde{\mathbb{G}}_{(m)}$, since it has a nontrivial characteristic module $C_{\Theta}$ (see ref. [12] and references therein). $C_{\Theta}$ is generated by $\tilde{X}_{B}^{L}$ and $\tilde{X}_{B}^{L}$, i.e., the left-invariant vector fields in the kernel of the Lie algebra cocycle. In particular, $\tilde{X}_{B}^{L}$ has integral curves obeying the classical equations of motion (Hamilton equations) except for the $\zeta$ co-ordinate equation which is responsible (in non-trivial physical systems) for the Bohr-Sommerfeld quantization rules [14]. The equations themselves require the identification of $B, \underline{A}, m \underline{V}$ with the physical variables $t, x, p$ respectively.

The quantization is finally realized as in the case of the Heisenberg-Weyl algebra except that now the polarization is enlarged by the characteristic subalgebra $\left\{\tilde{X}_{B}^{L}, \tilde{X}_{\underline{R}}^{L}\right\}$. Thus, the full (generalized) polarization is $\left\{\tilde{X}_{B}^{L}, \tilde{X}_{\underline{R}}^{L}, \tilde{X}_{\underline{A}}^{L}\right\}$ and the wave functions are complex functions on $\tilde{\mathbb{G}}_{(m)}$ such that

$$
\Xi . \Psi=i \Psi, \quad \tilde{X}_{\underline{A}}^{L} . \Psi=0, \quad \tilde{X}_{\underline{\underline{R}}}^{L} . \Psi=0, \quad \tilde{X}_{B}^{L} . \Psi=0 .
$$

In particular, the last equation in (2.5) is nothing other than the Schrödinger equation in momentum space

$$
i \hbar \partial \Psi / \partial t=p^{2} /(2 m) \Psi
$$

The $U(1)$ group law $\zeta^{\prime \prime}$ in (2.3) was expressly written so as to manifest the global (compact) structure of $U(1)$. Writting $\zeta$ locally as $\zeta=\exp i \chi / \hbar$, where the $U(1)$ local parameter $\chi$ admits dimension of an action, turns (2.3) into the group law for the central extension of $\mathbb{G}$ by the real line $R$. The last line in (2.3) is rewritten $\chi^{\prime \prime}=$ $\chi^{\prime}+\chi^{\prime}+m \xi\left(g^{\prime}, g\right)$. The quantization form (2.4) is now

$$
\Theta=\frac{1}{2} m[V \cdot d A-A \cdot d V]-\frac{1}{2} m V d B+d \chi
$$

and the equations of motion (the $\bar{X}_{B}^{L}$ generator) identify $\chi$ with the action of the system. The "wave" functions are now real $R$-equivariant functions $S$ on $\overline{\mathbb{G}}$ and the "wave" equation $\bar{X}_{B}^{L} . S=0$ turns out to be the Hamilton-Jacobi equation [14].

The example above provides the background for the formalism of quantization on a connected Lie group [12-15] whose essential ingredients we describe below.

Definition 2.1. (Connected) "Quantum" Group. A (connected) "quantum" group is a connected Lie group $\widetilde{G}$ which in turn is a right-principal bundle having as fibre an one-dimensional Lie subgroup $T(U(1)$ or $R)$.

We must remark that the compact structure of $T, T=U(1)$, will be responsible for the quantum nature of the associated physical system, while an open fibre, $T=R$, will lead to the classical description (Hamilton-Jacobi). The particular case where $\widetilde{G}$ is a central extension by $U(1)$ of a Lie group $G$ corresponds to a quantum system which admits a classical limit: the central extension of $G$ by the local group law (the additive group $R$ ) of $U(1)$. The classical limit then appears as a local version (from a local chart) of the quantum structure. As mentioned above the $\hbar$ 
constant is naturally removed from the classical group. On the other hand, a group $\tilde{G}$ whose group law breaks down when opening $U(1)$ reproduces the dynamics of a system without a classical limit.

Let $\theta^{L}$ be the canonical left-invariant one-form on $\tilde{G}$ [23] and $\Theta$ its vertical component [14] (see also ref. [10]), dual to the fundamental vector field $\tilde{X}_{(5)}^{L} \in$ $\mathscr{X}^{L}(\widetilde{G}) \approx \tilde{\mathscr{G}}$, where $\mathscr{X}^{L}(\widetilde{G})$ stands for the algebra of left-invariant vector fields on $\tilde{G}\left(X^{L}(\tilde{G})\right.$ generates the finite right action on $\tilde{G}$ on itself $)$. The one-form $\Theta$ will play the role of a connection form on the principal bundle $\widetilde{G}$ and its curvature $\Omega=d \Theta$ that of a presymplectic form. It can be proved [12] that in the particular case of a central extension the quotient of $(\tilde{G}, \Theta)$ by the characteristic module $C_{\Theta} \equiv$ $\left\{X \in \mathscr{X}(\widetilde{G}) / i_{X} \Theta=0=i_{X} d \Theta\right\}$ is a quantum manifold [15].

Definition 2.2. Characteristic Subalgebra and Evolution. The equations of "motion" are those associated with the left-invariant vector fields which generate the module $C_{\Theta}$.

It may easily be shown that $C_{\Theta}$ is generated as a free module by a set of left-invariant vector fields. These vector fields span an horizontal (with respect to $\Theta$ ) subalgebra $\mathscr{G}_{\Theta}$ of the Lie algebra $\widetilde{\mathscr{G}}$ of $\widetilde{G}$. The absolute time evolution is usually associated with one element in the centre of $\mathscr{G}_{\Theta}$.

Definition 2.3. Full Polarization. A full polarization, $\mathscr{P}$, is a maximal horizontal (with respect to $\Theta$ ) left subalgebra containing the characteristic subalgebra.

When the dimension of $\widetilde{G}$ is finite, Definition 2.3 eliminates the ambiguities of the standard definition of polarization $[16,27]$ in the geometric quantization approach. For infinite dimensional groups $\widetilde{G}$ Definition 2.3 seems to be quite adequate as it agrees with analogous structures (that of Borel subalgebras) of infinite-dimensional Lie algebras (see ref. [10] and references therein) which characterize the representations as well. Also the possibility exists of generalizing this definition allowing $\mathscr{P}$ to contain only a proper subalgebra of $\mathscr{G}_{\Theta}$. The existence of such a non-full polarization seems to be related to anomalies.

Definition 2.4. Wave Functions. We define wave functions as (complex) functions on $\widetilde{G}$ which satisfy

a) the condition of being $T$-function from the left,

$$
\Psi\left(g_{T} * g\right)=g_{T} * \Psi(g) \quad \forall g \in \widetilde{G}, \quad g_{T} \in T,
$$

i.e. $\Psi(\zeta g)=\zeta \Psi(g)$ for $\zeta \in U(1), \quad \Psi(g) \in C$ and $S(\chi+g)=\chi+S(g)$ for $\chi \in R$, $\Psi(g) \equiv S(g) \in R$.

b) the generalized Planck (or polarization) condition

$$
\tilde{X}^{L} . \Psi=0 \quad \forall \tilde{X}^{L} \in \mathscr{P} .
$$

In differential terms, a) reads

and

$$
\tilde{X}_{(\zeta)}^{R}, \Psi=i \Psi \text { for } \quad T=U(1)
$$

$$
\tilde{X}_{(\chi)}^{R} \cdot \Psi=1 \quad \text { for } \quad T=R,
$$

expressions which will decide the quantum or classical character of the physical operators. The Schrödinger equation just appears as one particular equation from 
those in (2.8). Condition a) above requires a further comment: only when the group $\widetilde{G}$ is a central extension by $U(1)$ or, rather, when $U(1)$ is a central subgroup, may (2.7) be written from the right, i.e. $\Psi\left(g * g_{T}\right)=g_{T} * \Psi(g) \equiv \Psi(g) * g_{T}$, thus reproducing the ordinary $U(1)$-function condition on a right principal bundle. Thus, when $U(1)$ is not central the parallelism with the geometric quantization approach is in this respect broken.

Definition 2.5. Dynamical Operators. The dynamical operators are the rightinvariant vector fields on $\tilde{G}, \mathscr{X}^{R}(\tilde{G})$, acting on the wave functions as ordinary derivations.

Note that the action of $\mathscr{X}^{R}(\tilde{G})$ is compatible with the polarization conditions since the right- and left-invariant vector fields commute. The $T$-function condition, nevertheless, could restrict the set of true operators to a subalgebra of $\mathscr{X}^{R}(\widetilde{G})$ in the case where $T$ is not central (see Sect. III where this question is analyzed further).

The formalism now ends with the definition of a hermitian product which makes the space of wave functions $\mathscr{H}(\tilde{G})$ a prehilbertian space. The definition of the hermitian product, with respect to which the operators generate (pseudo-) unitary transformations, requires the use of an invariant measure (unitarity appears for determinate values of the parameters in the theory, for instance, cohomology parameters for centrally extended groups). It is obtained from the left-invariant forms on $\widetilde{G}[14]$.

\section{Algebraic Quantization on a Group}

The use of discrete symmetry transformations inside the quantum group $\widetilde{G}$ requires a generalization of the geometric formalism briefly described in the previous section. Indeed, in the previous formalism all restrictions on the wave functions, i.e., the $T$-function condition and the generalized Planck condition, were imposed through Lie derivatives defined by infinitesimal generators of the group action. Moreover, the definition of the polarization $\mathscr{P}$ itself made use of a differential form, $\Theta$, intimately related to the differential system $\mathscr{X}^{L}(\widetilde{G})$. Thus, we have to start by reinterpreting in finite or integral form the $T$-function condition (it was already given in finite form [(2.7)] and the generalized Planck condition (Definition 2.4b)). Specifically, the polarization condition will now read

$$
\Psi\left(g * G_{\mathscr{P}}\right)=\Psi(g),
$$

where $G_{\mathscr{p}}$ will be the subgroup generalizing the transformations generated by the left-invariant vector field of $\mathscr{P}$ in the previous formalism. In particular, what was previously the characteristic module associated with $\Theta, C_{\Theta}$, is now replaced by a subgroup of $\tilde{G}$ algebraically characterized without any reference to a differential form. We shall label this group $G_{C}$.

To seek an algebraic characterization of $G_{C}$ we note that $C_{\Theta}$ might have been defined as the maximal horizontal subalgebra. $\mathscr{G}_{C_{\Theta}}$, such that $\left[\mathscr{G}_{C_{\Theta}}, \mathscr{X}^{L}(\tilde{G})\right]$ is horizontal. In fact, making use of the Maurer-Cartan equations one can write, on left-invariant vector field ( $\Theta$ is also left-invariant),

$$
d \Theta(X, Y)=-\Theta([X, Y]),
$$


so that one has $\Theta([X, Y])=0 \forall X \in \mathscr{G}_{C_{\Theta}}, Y \in \mathscr{X}^{L}(\widetilde{G})$, because $i_{X} d \Theta=0=i_{X} \Theta$. Finally, the horizontality condition can also be replaced by an algebraic one given in group terms rather than using Lie algebra generators. Labelling $G_{C_{\Theta}}$ the characteristic subgroup having $\mathscr{G}_{C_{\Theta}}$ as its Lie algebra, the horizontality condition for $\left[\mathscr{G}_{C_{\Theta}}, \mathscr{X}^{L}(\tilde{G})\right]$ is replaced by

$$
\left[G_{C_{\Theta}}, \tilde{G}\right] \cap T=1_{\tilde{G}},
$$

where [,] now stands for the group commutator $\left(\left[g^{\prime}, g\right]=g^{\prime} g g^{\prime-1} g^{-1}\right)$. Thus, (3.3) constitutes the algebraic characterization of $G_{C_{\Theta}}$ for the case of a connected Lie group $\tilde{G}$.

The same algebraic condition (3.3), but with $G_{C}$ replacing $G_{C_{\Theta}}$, along with the algebraic version (3.1) of the polarization condition provides the generalization of the geometric formalism needed for the incorporation of discrete operations. However another generalization is required if the structural group $T$ is to be larger than $U(1)$ or $R$ and especially if $T$ is nonabelian and therefore noncentral. $T$ in (3.3) must be replaced by the one-dimensional $(U(1)$ or $R$ ) subgroup of $T$ to which it generalizes. In general $T$ will be, in turn, a principal bundle (perhaps trivial) having $T_{0} \equiv U(1)$ or $R$ as the structure group. In this case it is the structure group of the enlarged group $T$ that must be put in (3.3).

A consequence which follows from the noncentral character of $T$ and from the fact that wave functions must obey the $T$-function as well as the Planck conditions is that the prehilbertian space $\mathscr{H}(\widetilde{G})$ will not be stable under the whole set of (finite) left transformations (generated by right-invariant vector fields when $\widetilde{G}$ is a connected Lie group). This will lead us to select a preferred class of left translations defining "good" operators.

Moreover, it has to be realized that if $T$ is larger than $U(1)$ or $R$ a representation space bigger than $C$ is also needed and that in general more than one representation of $T$ may be utilized in the $T$-function condition. In this case non-equivalent quantizations will correspond to each of the representations of $T$.

Finally we must point out that a $T$-function condition by the whole group $T$ might be in general too strong for some groups $\widetilde{G}$ and that some sort of polarization subgroup $T_{p}$ of $T$ should be used. This actually happens when an "anomaly" is present in the Lie algebra of $T$ or, in general, when some of the parameters in $T$ play the role of co-ordinate-momentum pairs. The group $T$ itself is, in this case, a quantum group and the maximum set of compatible constraints that can be in general imposed on any representation is indeed a polarization. As a general rule, the complete constraint subgroup $T$ can be imposed on the wave function only if $T / T_{0}$ is a subgroup of $G_{\mathscr{g}}$. On the other hand, if $G_{\mathscr{p} p}$ contains only a polarization subgroup $T_{p}^{\prime}$ of $T$, a polarization subgroup $T_{p}$ of $T$, dual to $T_{p}^{\prime}$ must replace $T$ when writing down the $T$-function condition.

After this rather detailed justification of the algebraic quantization structure we now give a more concise account.

Definition 3.1. "Quantum" Group. A "quantum" group is a.(Lie ?) group $\tilde{G}$ which in turn is a right-principal bundle with structure group $T$.

We will think of $T$ as containing a one-dimensional subgroup $T_{0}(U(1)$ or $R)$. 
Indeed, $T$ will be a principal bundle having $T_{0}$ as the structure group. Of course, the principal structures we are referring to are defined in algebraic (as opposed to differential) terms.

Definition 3.2. Characteristic Subgroup and Evolution. A characteristic subgroup $G_{C}$ is a maximal subgroup of $\widetilde{G}$ such that

$$
\left[G_{C}, \tilde{G}\right] \cap T_{0}=1_{\tilde{G}}, \quad G_{C} \cap T_{0}=1_{\tilde{G}} .
$$

The right translations by elements in $G_{C}$ are the generalized evolution transformations in terms of which the generalized equations of motion will be given as a part of the finite polarization conditions that follow. The finite Schrödinger equation will be associated with an element in the centre of $G_{C}$.

Definition 3.3. Full Polarization. A full polarization $G_{\mathscr{P}}$ is a maximal subgroup of $\widetilde{G}$ such that (see comments after Definition 2.3)

$$
G_{C} \subset G_{\mathscr{P}}, \quad G_{\mathscr{P}} \cap T_{0}=1_{\tilde{G}} .
$$

Let $T_{p}$ be a polarization subgroup of $T$, i.e., $T_{p}$ is defined with respect to $T$ just as $G_{\mathscr{P}}$ was with respect to $\tilde{G}$. Let us consider the subgroup $T_{B}$ generated by $T_{p} \cup T_{0}$ which generalizes the notion of a Borel subgroup [29].

Definition 3.4. Wave Functions. A wave function is a function $\Psi$ on $\widetilde{G}$, taking its values on a vector space $E$, such that

a) $\Psi$ is a $T_{B}$-function from the left

$$
\Psi\left(g_{T_{B}} * g\right)=D\left(g_{T_{B}}\right) \Psi(g),
$$

where $D$ is a representation of $T_{B}$ on $E$ which characterizes the quantization.

b) $\Psi$ satisfies the generalized Planck condition

$$
\Psi\left(g * G_{g p}\right)=\Psi(g) .
$$

The space of wave functions is labelled $\mathscr{H}(\widetilde{G})$.

In Definition 3.4, $D\left(g_{T_{B}}\right)$ generalizes the natural representation of $U(1)$ on the complex numbers $C$ where $\Psi$ takes its values. Now the wave functions take values on the carrier space $E$ for the concrete representation $D$. In turn, this carrier space could be obtained by using the group quantization formalism again but now with $T$ as the starting point. In this way the whole procedure remains quite canonical (an analogous situation appears in induced representation theory when the little group of an orbit is again a semidirect product of two groups).

Definition 3.5. "Dynamical" and "good" Operators. We shall name "Dynamical" operators the left translations $L_{g}, g \in \widetilde{G}$ acting from the left on the arguments of the functions on $\tilde{G}$. The subgroup $H_{\mathscr{H}} \subset \widetilde{G}$ of dynamical transformations which are compatible with (3.6) and (3.7) will be called the subgroup of "good" operators; $G_{\mathscr{H}}$ is the maximal subgroup which fulfills

$$
\operatorname{Ad}(\widetilde{G})\left[T_{B}, G_{\mathscr{H}}\right] \subset G_{\mathscr{P} P}
$$

We will see in the examples of Sect. IV and V that the set of dynamical operators also makes physical sense even though it only preserves the space of $T_{0}$-functions. 
Finally we note that an invariant (from the left) scalar product can be defined in the form

$$
(\Psi, \Phi)=\sum_{\widetilde{G}} \mu(g) \Psi^{*}(g) \Phi(g)
$$

if the representation $D$ of $T_{B}$ is unitary. Of course, $\sum_{\widetilde{G}} \mu$ is a generalized (discretecontinuous) left-invariant measure on $\tilde{G}$ (see Ref. [11] for the continuous case).

\section{The System of $\mathbf{n}$ Identical Particles}

One of the most significant modern geometric quantization formalisms is that of Souriau and Kostant [16,27] (see also Ref. [28]) which predicts the existence of two inequivalent quantum manifolds on the symplectic manifold $M$ of solutions associated with a system of $n$ identical particles [16], i.e., of as many inequivalent quantum manifolds as there are elements in the dual group of the homotopy group (the permutation group $S_{n}$ ) of the manifold $M$. Nevertheless, the explicit construction (which is not given in the original papers) is quite involved. Indeed, the space of solutions of a system of $n$ identical particles is mathematically realized by taking the quotient by $S_{n}$ of the product of $n$ manifolds identical to the manifold of solutions of one particle, and it turns out to be a manifold only for dim $M \leqslant 2$. For $\operatorname{dim} \geqslant 3$ the additional operation of eliminating the diagonal from $M \times M \times \cdots \times M$ is required, a procedure which leads to rather involved manifolds for $n \geqslant 4$ [30]. For $n=2$ and $M=R^{m}$ with $m \geqslant 3$ one finds $R^{m} \times p^{m-1}(R) \times R$.

In our algebraic formalism the quantization of $n$ identical particles moving in an $m$-dimensional space can be achieved, without subtracting any submanifold, for arbitrary $n$ and $m$. We shall limit the explicit calculations to the case of two identical particles moving in $R^{3}$ although the group law for $n$ of them as well as a few comments on this general case will be given at the end of this section.

For two particles the problem is essentially reduced to that of one (reduced) particle whose inversion with respect to the origin (centre of mass) is given by the interchange operation between both particles. Our (non-connected) group law is then (we put $\hbar=1$ )

$$
\begin{aligned}
& B^{\prime \prime}=B^{\prime}+B, \\
& \underline{A}^{\prime \prime}=\underline{A}^{\prime}+\underline{R}^{\prime} \pi^{\prime} \underline{A}+\underline{V}^{\prime} B, \\
& \underline{V}^{\prime \prime}=\underline{V}^{\prime}+\underline{R}^{\prime} \pi^{\prime} \underline{V}, \\
& \underline{R}^{\prime \prime}=\underline{R^{\prime}} \underline{R}, \\
& \zeta^{\prime \prime}=\zeta^{\prime} \zeta \exp i \mu\left\{\underline{A}^{\prime} \cdot \underline{R}^{\prime} \pi^{\prime} \underline{V}+B\left(\underline{V}^{\prime} \cdot R^{\prime} \pi^{\prime} \underline{V}+1 / 2 \underline{V}^{\prime 2}\right)\right\}, \\
& \pi^{\prime \prime}=\pi^{\prime} \pi,
\end{aligned}
$$

where $B, \underline{A}, \underline{V}, \underline{R}$ are similar to the Galilean parameters given in (2.3), $\mu$ stands for the reduced mass and $\pi \in Z_{2}$, i.e., either the identity or the exchange operation.

Although (4.1) corresponds in fact to a central $U(1)$-extension $\widetilde{G} I_{(\mu)}$ of a Galilei group $\mathbb{G} I$ in which $O(3)$ replaces $S O(3)$, the structure we are interested in is that of a right principal bundle on the Galilei group $\mathbb{G}$ with fibre $T \equiv U(1) \times Z_{2}$. We shall name this structure $\widetilde{G}\left(\mathbb{G}, U(1) \times Z_{2}\right)$. 
A crucial calculation in what follows is the group commutator of two arbitrary elements. Writing the generic element $g$ as $(B, \underline{A}, \underline{V}, \underline{R} ; \zeta, \pi)$ we get

$$
\begin{aligned}
{\left[g^{\prime}, g\right]=} & \left(0,\left(1-\operatorname{Ad}\left(\underline{R}^{\prime}\right)(\underline{R}) \pi\right)\left(\underline{A}^{\prime}-\underline{V}^{\prime} B^{\prime}\right)+\left(\underline{R}^{\prime} \pi^{\prime}-[\underline{R}, R]\right)\left(\underline{A}-\underline{V} B^{\prime}\right)\right. \\
& +\operatorname{Ad}\left(\underline{R}^{\prime}\right)(\underline{R}) \pi B \underline{V}^{\prime}-\underline{R}^{\prime} \pi^{\prime} B^{\prime} \underline{V},\left(1-\operatorname{Ad}\left(\underline{R^{\prime}}\right)(\underline{R}) \pi\right) \underline{V} \\
& +\left(\underline{R}^{\prime} \pi^{\prime}-\left[\underline{R}, \underline{R}^{\prime}\right]\right) \underline{V},\left[\underline{R}^{\prime}, \underline{R}\right] ; \exp i \mu\left\{\xi\left(g^{\prime}, g\right)-\xi\left(g^{\prime-1}, g^{\prime}\right)\right. \\
& \left.\left.-\xi\left(g^{-1}, g\right)+\xi\left(g^{\prime-1}, g^{-1}\right)+\xi\left(g^{\prime} g, g^{\prime-1} g^{-1}\right)\right\}, 1\right),
\end{aligned}
$$

where $\xi\left(g^{\prime}, g\right)$ - the $\mathbb{G} I$ cocycle - is $1 /(i \mu)$ times the exponent appearing in (4.1). The rather complicated term $\xi\left(g^{\prime} g, g^{-1} g^{-1}\right)$ is given by

$$
\begin{aligned}
- & \left(\underline{A}^{\prime}+\underline{R}^{\prime} \pi^{\prime} \underline{A}+\underline{V}^{\prime} B\right) \cdot\left[\underline{R^{\prime}}, \underline{R}\right]\left(\underline{V}+\underline{R} \pi \underline{V}^{\prime}\right)+\left(B^{\prime}+B\right)\left(\left(\underline{V}^{\prime}+\underline{R}^{\prime} \pi^{\prime} \underline{V}\right)\right. \\
& \left.\cdot\left[\underline{R}^{\prime}, \underline{R}\right]\left(\underline{V}+\underline{R} \pi \underline{V}^{\prime}\right)-1 / 2\left(\underline{V}^{\prime}+\underline{R}^{\prime} \pi^{\prime} \underline{V}\right)^{2}\right) .
\end{aligned}
$$

A direct calculation provides $G_{C}$ and $G_{\mathscr{P}}$ :

$$
\begin{aligned}
& G_{C}=\{\text { Time trans. }\} \cup\{S O(3)\} \cup Z_{2} \\
& G_{\mathscr{P}}=G_{C} \cup\{\text { Space transl. }\} .
\end{aligned}
$$

The wave functions are complex functions on $\widetilde{G}\left(\mathbb{G}, U(1) \times Z_{2}\right)$ which satisfy the $\left(U(1) \times Z_{2}\right)$-function condition and the generalized Planck condition with respect to the subgroup $G$. The $T$-function condition reads (in this case $T_{B}$ and $T$ coincide)

$$
\Psi\left(\left(\zeta^{\prime}, \pi^{\prime}\right) *(B, \underline{A}, \underline{V}, \underline{R} ; \zeta, \pi)\right)=D\left(\zeta^{\prime}, \pi^{\prime}\right) \Psi(B, \underline{A}, \underline{V}, \underline{R} ; \zeta, \pi)
$$

where $D$ stands for any one of the possible representations of $T$ on $C$; they are parametrized by $2 s, n \in Z^{+}$,

$$
D(\zeta, \pi)=\pi^{2 s \zeta n} .
$$

We shall restrict ourselves - as it was in fact done in the previous formalism - to the case $n=1$ ( $n>1$ would lead, in general, to vector subspaces of $\mathscr{H}(\tilde{G})$ for $n=1)$.

The full polarization condition for an arbitrary element $g$ of $G_{\mathscr{P}}$ is written

$$
\begin{aligned}
\Psi\left(B^{\prime}, \underline{A}^{\prime}, \underline{V}^{\prime}, \underline{R}^{\prime} ; \zeta^{\prime}, \pi^{\prime}\right)= & \Psi\left(B^{\prime}+B, \underline{A}^{\prime}+\pi^{\prime} \underline{R}^{\prime} \underline{A}+\underline{V}^{\prime} B, \underline{V^{\prime}}, \underline{R}^{\prime} \underline{R} ;\right. \\
& \left.\zeta^{\prime} \exp i \mu\left(B \underline{V}^{\prime 2} / 2\right) ; \pi^{\prime} \pi\right), \quad \forall g^{\prime} \in \widetilde{G},
\end{aligned}
$$

and choosing for $g$ the particular value $B=-B^{\prime}, \underline{A}=-\pi^{\prime} \underline{R}^{\prime-1} \times\left(A^{\prime}-\underline{V}^{\prime} B^{\prime}\right)$, $\underline{R}=\underline{R}^{\prime-1}, \pi=\pi^{\prime}$, one gets

$$
\Psi(B, \underline{A}, \underline{V}, \underline{R} ; \zeta, \pi)=\Psi\left(0, \underline{0}, \underline{V}, \underline{1} ; \zeta \exp -i \mu\left(B \underline{V}^{2} / 2\right), 1\right),
$$

where the primes have been dropped everywhere. Using simultaneously (4.4), (4.5) and (4.6) (for the case $n=1$ ) the wave functions become

$$
\Psi(B, \underline{A}, \underline{V}, \underline{R} ; \zeta, \pi)=\zeta \exp \left(-i \mu B \underline{V}^{2} / 2\right) \Phi(\underline{V}),
$$

where $\Phi(\underline{V})$ is a function depending only on $\underline{V}$ and satisfying

$$
\Phi(\underline{V})=\pi^{2 s} \Phi(\pi \underline{V}) .
$$

Equation (4.8) provides the expected characterization of either Bose and Fermi momentum wave functions depending on whether $s$ is integer or half-integer. 
To find the subgroup whose left-translations leave $\mathscr{H}(\tilde{G})$ stable we must solve Eq. (3.8). To do this, we first observe that if " $a$ " is a rotation, time translation or inversion one has $[T, a]=1_{\tilde{G}}$, and thus $\operatorname{Ad}(g)[T, a]=1_{\tilde{G}}$. On the other hand, if " $a$ " is a spatial translation $\left(\underline{A}^{\prime}\right)$ or a boost $\left(\underline{V}^{\prime}\right)$ its commutator with the inversion is another spatial translation or another boost. Thus, calculating $\operatorname{Ad}(g)\left(\underline{A}^{\prime}\right)$ and $\operatorname{Ad}(g)\left(\underline{V}^{\prime}\right) \forall g$, we get

$$
\begin{aligned}
& \operatorname{Ad}(g)\left(\underline{A}^{\prime}\right)=\left(0, \pi \underline{R} \underline{A}^{\prime}, \underline{0}, \underline{I} ; \exp i \mu\left(\pi \underline{A^{\prime}} \underline{R} \underline{V}\right), 1\right) \notin \tilde{G}, \\
& \operatorname{Ad}(g)\left(\underline{V}^{\prime}\right)=\left(0, \underline{0}, \pi \underline{R} \underline{V}^{\prime}, \underline{I} ; \exp i \mu\left(\underline{A} \underline{R} \pi \underline{V}^{\prime}-B \underline{V}^{\prime 2} / 2\right), 1\right) \notin \widetilde{G}_{\mathscr{P}} .
\end{aligned}
$$

Accordingly $G_{\mathscr{H}}=\left\{Z_{2}\right\} \cup\{\mathrm{SO}(3)\} \cup\{$ Time transl. $\}$. The corresponding (good) operators, i.e., the right generators (up to a constant factor $i$ and putting $\hbar=1$ ) of the continuous transformations on $G_{\mathscr{H}}$ and the discrete nontrivial operator of $Z_{2}$, have the following action ( $\underline{\varepsilon}$ is the $\mathrm{SO}(3)$ parameter)

$$
\begin{aligned}
i \tilde{X}_{(B)}^{R} \cdot \Phi(\underline{V}) & =1 / 2 \mu \underline{V}^{2} \Phi(\underline{V}), \\
i \tilde{X}_{(\varepsilon)}^{R} \cdot \Phi(\underline{V}) & =-i \underline{V} \wedge \partial / \partial \underline{V} \Phi(\underline{V}), \\
\Pi \Phi(\underline{V}) & =(-1)^{2 s} \Phi(\underline{V}) .
\end{aligned}
$$

We end this section with the generalization of the group law (4.1) to the case of $n$ identical particles. Disregarding rotations the group law can be written as follows:

$$
\begin{aligned}
B^{\prime \prime} & =B^{\prime}+B \\
A_{i}^{\prime \prime} & =A_{i}^{\prime}+A_{\pi^{\prime-1}(i)}+V_{i}^{\prime} B, \\
V_{i}^{\prime \prime} & =V_{i}^{\prime}+V_{\pi^{\prime}-1}(i) \\
\zeta^{\prime \prime} & =\zeta^{\prime} \zeta \exp i m \sum_{i}\left\{A_{i}^{\prime} V_{\pi^{\prime-1}(i)}+B\left(V_{i}^{\prime} V_{\pi^{\prime-1}(i)}+\frac{1}{2}\left(V_{i}\right)^{2}\right\}\right. \\
\pi^{\prime \prime} & =\pi^{\prime} \pi,
\end{aligned}
$$

where $\pi$ now runs over the permutation group $S_{n}$. The entire formalism can be followed step by step with the natural generalization of the one-dimensional representations (4.5) which must now be rewritten

$$
D(\zeta, \pi)=\operatorname{sign}(\pi)^{2 s \zeta^{n}} .
$$

Nevertheless, the group $S_{n}$ is now non-abelian and therefore additional irreducible representations of dimension greater than one can be considered as well. The corresponding wave functions will have the symmetry of some Young tableau. The analysis of these new quantizations and their connection with parastatistics [31] deserves a separate study.

\section{The Principal Bundle Virasoro (s) \{Affine $\boldsymbol{\Omega S U}(2)\} \rightarrow \boldsymbol{\Omega S U}(\mathbf{2})$}

We now consider a typical case of nonabelian constraints: the Virasoro constraints $L_{n \geq 0} \Psi=0$ that the wave functions of strings (on a group manifold) must obey $[21,22]$. These constraints will appear as $T_{B}$-function conditions from a concrete principal bundle group whose fibre contains the whole Virasoro group. As no 
discrete transformation is considered we may think of invariant vector fields, Lie algebra structure etc. Thus, for instance, Lie commutators of the (say) rightinvariant vector fields of the semidirect product of the $U(1)$-extended Virasoro group and the Affine $S U(2)$ loop group [11] are

$$
\begin{aligned}
{\left[\tilde{X}_{z_{q}}^{R}, \tilde{X}_{z_{p}^{*}}^{R}\right] } & =-2 i \tilde{X}_{\theta_{q+p}}^{R}-2(\alpha q+i N / 2) \delta_{q,-p} \tilde{X}_{\zeta}^{R}, \\
{\left[\tilde{X}_{\theta_{q}}^{R}, \tilde{X}_{z_{p}}^{R}\right] } & =-i \tilde{X}_{z_{q+p},}^{R},\left[\tilde{X}_{\theta_{q}}^{R}, \tilde{X}_{z_{p}^{*}}^{R}\right]=i \tilde{X}_{z_{q}^{*}+p}^{R}, \\
{\left[\tilde{X}_{\theta_{q}}^{R}, \tilde{X}_{\theta_{p}}^{R}\right] } & =-\alpha q \delta_{q,-p} \tilde{X}_{\zeta}^{R} \\
{\left[\tilde{X}_{l_{n}}^{R}, \tilde{X}_{l_{m}}^{R}\right] } & =(n-m) \tilde{X}_{l_{n+m}}^{R}+1 / 12\left(c n^{3}-c^{\prime} n\right) \delta_{n,-m} \tilde{X}_{\zeta}^{R}, \\
{\left[\tilde{X}_{l_{n}}^{R}, \tilde{X}_{z_{m}}^{R}\right] } & =-m \tilde{X}_{z_{n+m},}^{R} \quad\left[\tilde{X}_{l_{n}}^{R}, \tilde{X}_{z_{m}^{*}}^{R}\right]=-m \tilde{X}_{z_{n+m}^{*}}^{R}, \\
{\left[\tilde{X}_{l_{n}}^{R}, \tilde{X}_{\theta_{m}}^{R}\right] } & =-m \tilde{X}_{\theta_{n+m}}^{R}-m N / 2 \delta_{n,-m} \tilde{X}_{\zeta}^{R}, \\
{\left[\tilde{X}_{\zeta}^{R}, \text { any other }\right] } & =0
\end{aligned}
$$

where $z_{m}, z_{n}^{*}, \theta_{p}, m, n, p \in Z$, parametrize $\Omega S U(2)\left(z_{0}, z_{0}^{*}, \theta_{0}\right.$ parametrize $S U(2)$, $\theta_{0}$ the Cartan subgroup), $l_{m}, m \in Z$ parametrize the Virasoro group, and $\zeta=\exp i \phi$ the central $U(1)$ subgroup. The algebra (5.1) was already considered in ref. [11] where all possible quantizations associated with the $U(1)$ central extension structure of the group Virasoro (5) AAffine $\Omega S U(2)$ \} were classified. These quantizations correspond, in particular, to those which could be obtained by using the standard geometric quantization methods on co-adjoint orbits [32]. Note that the fibration we now are interested in, i.e., Virasoro (5) Affine $\Omega S U(2)\} \rightarrow \Omega S U(2)$ is different and has, in turn, the non-abelian $U(1)$-centrally-extended Virasoro group as the structure group.

To illustrate how the Lie algebra (5.1) in fact corresponds to a Lie group which in turn is a principal bundle having the Virasoro group within its fibre, let us write down the explicit group law from which the algebra (5.1) can be derived. Up to fourth order the group law is as follows:

$$
\begin{aligned}
z_{n}^{\prime \prime}= & z_{n}^{\prime}+z_{n}-i z_{n-m}^{\prime} \theta_{m}+(n-m) l_{m}^{\prime} z_{n-m}-z_{n-m-p}^{\prime} z_{m} z_{p}^{*}-z_{n-m-p}^{\prime *} z_{m} z_{p} \\
& -1 / 2 z_{n-m-p}^{\prime} \theta_{m} \theta_{p}-z_{n-m-p}^{\prime} z_{m}^{* \prime} z_{p}-i(m-p) l_{p}^{\prime} z_{n-m}^{\prime} \theta_{m-p} \\
& +1 / 2\left(n-m_{1}\right)\left(n-m_{1}-m_{2}\right) l_{m_{1}}^{\prime} l_{m 2}^{\prime} z_{n-m_{1}-m_{2}}+\cdots, \\
z_{n}^{* \prime \prime}= & z_{n}^{* \prime}+z_{n}^{*}+i z_{n-m}^{* \prime} \theta_{m}+(n-m) l_{m}^{\prime} z_{n-m}^{*}-z_{n-m-p}^{* \prime} z_{m}^{*} z_{p}-z_{n-m-p}^{\prime} z_{m}^{*} z_{p}^{*} \\
& -1 / 2 z_{n-m-p}^{* \prime} \theta_{m} \theta_{p}-z_{n-m-p}^{* \prime} z_{m}^{\prime} z_{p}^{*}+i(m-p) l_{p}^{\prime} z_{n-m}^{* \prime} \theta_{m-p} \\
& +1 / 2\left(n-m_{1}\right)\left(n-m_{1}-m_{2}\right) l_{m_{1}}^{\prime} l_{m 2}^{\prime} z_{n-m_{1}-m_{2}}^{*}+\cdots, \\
\theta_{n}^{\prime \prime}= & \theta_{n}^{\prime}+\theta_{n}+(n-m) l_{m}^{\prime} \theta_{n-m}+i\left(z_{n-m}^{\prime} z_{m}^{*}-z_{n-m}^{* \prime} z_{m}\right) \\
& +i(m-p) l_{p}^{\prime}\left(z_{n-m}^{\prime} z_{m-p}^{*}-z_{n-m}^{* \prime} z_{m-p}\right)+\left(z_{n-m-p}^{\prime} z_{m}^{*}+z_{n-m-p}^{* \prime} z_{m}\right) \theta_{p} \\
& +1 / 2\left(n-m_{1}\right)\left(n-m_{1}-m_{2}\right) l_{m_{1}}^{\prime} l_{m 2}^{\prime} \theta_{n-m_{1}-m_{2}}+\cdots, \\
l_{n}^{\prime \prime}= & l_{n}^{\prime}+m l_{m} l_{n-m}^{\prime}+1 / 2 ! m^{2} l_{m} l_{p} l_{n-m-p}^{\prime}+\cdots, \\
\phi^{\prime \prime}= & \phi^{\prime}+\phi+\alpha \xi_{\alpha}+N \xi_{N}+c \xi_{c}+c^{\prime} \xi_{c},
\end{aligned}
$$




$$
\begin{aligned}
\xi_{\alpha} \equiv & 1 / 2\left[n\left(2 z_{n}^{* \prime} z_{-n}+2 z_{n}^{\prime} z_{-n}^{*}+\theta_{n}^{\prime} \theta_{-n}\right)+i(m-n)\left(z_{n}^{\prime} z_{m}^{*} \theta_{-n-m}\right.\right. \\
& \left.-z_{n}^{* \prime} z_{m} \theta_{-n-m}\right)+i\left(\theta_{n}^{\prime} z_{m}^{\prime} z_{-n-m}^{*}-\theta_{n}^{\prime} z_{m}^{* \prime} z_{-n-m}\right) \\
& \left.+n(n+p) l_{p}^{\prime}\left(2 z_{n}^{* \prime} z_{-n-p}+2 z_{n}^{\prime} z_{-n-p}^{*}+\theta_{n}^{\prime} \theta_{-n-p}\right)+\cdots\right], \\
\xi_{N}= & 1 / 2\left(\theta_{0}^{\prime \prime}-\theta_{0}^{\prime}-\theta_{0}\right), \\
\xi_{c}= & -1 / 2\left[n^{3} l_{-n} l_{n}^{\prime}+1 / 2 ! n m(n+m)^{2} l_{-n-m}^{\prime} l_{n} l_{m}\right. \\
& \left.-1 / 2 !(n+m)^{2}\left(n^{2}+m^{2}+n m\right) l_{n}^{\prime} l_{m}^{\prime} l_{-n-m}+\cdots\right], \\
\xi_{c^{\prime}}= & 1 / 2\left(l_{0}^{\prime \prime}-l_{0}^{\prime}-l_{0}\right) .
\end{aligned}
$$

It is clear from (5.2) that the Virasoro group only acts from the left (we write a generic group law as $g^{\prime \prime}=g^{\prime} * g$ ) on $\Omega S U(2)$; the non-primed $l_{n}$ parameters only appear in the fibre $\left(l_{n}^{\prime \prime}, \phi^{\prime \prime}\right)$ group law.

Following our general prescriptions for obtaining quantum wave functions we start with functions on $\tilde{G}=$ Virasoro (S) $\{$ Affine $S U(2)\}$ restricted by one set of full polarization conditions. For instance, let us choose the full polarization which generalizes that used in ref. [11] to calculate the standard representations of Affine $\Omega S U(2)$, i.e.,

$$
\mathscr{P}_{A}^{\text {Standard }} \equiv\left\langle\tilde{X}_{\theta_{n} \leq 0}^{L}, \tilde{X}_{z_{q \leq 0}}^{L}, \tilde{X}_{z_{q}^{*}<0}^{L}, \tilde{X}_{l_{m \leq 0}}^{L}\right\rangle
$$

The generalized Planck conditions $\mathscr{P}_{A}^{\text {Standard }} \cdot \Psi=0$ must be imposed in addition to the $T_{B}$-function conditions. Here $T$ is the $U(1)$-extended Virasoro subgroup and $T_{B}$ can be chosen as generated by

$$
\left\langle Z_{\zeta}, Z_{l_{m \geq 0}}\right\rangle \subset \text { Lie algebra of } T .
$$

With the representation $D$ of $T_{B}$ characterized by

$$
d D\left(Z_{\zeta}\right)=i, \quad d D\left(Z_{l_{m}}\right)=a \delta_{m, 0},
$$

the $T_{B}$-function conditions read

$$
\begin{gathered}
\tilde{X}_{\zeta}^{R} \cdot \Psi=i \Psi, \\
\tilde{X}_{l_{m \geq 0}^{R}}^{R} . \Psi=a \delta_{m, 0} \Psi .
\end{gathered}
$$

Note that taking the dual polarization $\left(Z_{l_{m \leq 0}}\right)$ leads to a trivial result.

To prove finally that $(5.6 \mathrm{~b})$ are in fact the ordinary constraints imposed in string theory let us restrict ourselves to the subgroup $\left\{l_{0}\right\}$ (S) Affine $\left.\Omega S U(2)\right\}$ whose wave functions are known [11]. In this case (5.3) reduces to

$$
\left\langle\tilde{X}_{\theta_{n \leq 0}}^{L}, \tilde{X}_{z_{q \leq 0}}^{L}, \tilde{X}_{z_{q}^{*}<0}^{L}, \tilde{X}_{l_{0}}^{L}\right\rangle
$$

and (5.6) to

$$
\begin{aligned}
& \tilde{X}_{\zeta}^{R} \cdot \Psi=i \Psi \\
& \tilde{X}_{l_{0}}^{R} \cdot \Psi=a \delta_{m, 0} \Psi
\end{aligned}
$$

(5.8a) is nothing other than our ordinary $U(1)$-function condition and $\tilde{X}_{l_{0}}^{R}$ is just the Sugawara construction of the $L_{0}$ operator. An explicit calculation of the wave functions on the whole Virasoro (S) Affine $\Omega S U(2)\}$ will be presented elsewhere. 
We will see how the Virasoro group can be realized with or without anomaly $\left(c=0=c^{\prime}\right)$ although in the latter case on wave functions depending on the Virasoro group parameters $l_{n}$ as additional variables playing the role of bosonic ghosts.

As a last comment we want to point out the meaning of the subgroup $G_{\mathscr{H}}$ of "good operators" in the present example. Although the actual solution of (3.8) is nothing other than the $S U(2)$ subgroup, a solution of (3.8) (or rather its infinitesimal counterpart) inside the enveloping algebra of $\widetilde{G}$ would contain the DDF operators [33]. An alternative possibility of incorporating the DDF set into the scheme might be found in the theory of hyperbolic Kac-Moody algebras [34]. A formal hyperbolic Kac-Moody group should provide a subgroup $G_{\mathscr{H}}$ containing the DDF algebra.

Acknowledgements. One of us (V.A.) wishes to thank C. J. Isham for his hospitality at Imperial College. We all are grateful to him for very valuable comments and suggestions and for reading the manuscript.

\section{References}

1. Ne'eman, Y., Regge, T.: Gauge theory of gravity and supergravity on a group manifold. Riv. Nuovo Cim. 1, fasc. 5 (1978)

2. Witten, E.: Commun. Math. Phys. 92, 455 (1984)

3. Knizhnik, V. G., Zamolodchikov, A. B.: Nucl. Phys. B247, 83 (1984)

4. Nemeschansky, D., Yankielowicz, S.: Phys. Lett. 54, 628 (1985)

5. Gepner, D., Witten, E.: Nucl. Phys. B278, 493 (1986)

6. Mickelsson, J.: CTP preprint \# 1448

7. Isham, C. J., Kakas, A. C.: Class. Quant. Grav. 1, 621 (1984) and 1, 633 (1984)

8. Bakas, I., Kakas, A. C.: J. Phys. A20, 3713 (1987)

9. Bakas, I., Kakas, A. C.: Class. Quant. Grav. 4, 67 (1987)

10. Aldaya, V., Navarro-Salas, J.: Commun. Math. Phys. 113, 375 (1987)

11. Aldaya, V., Navarro-Salas, J.: Lett. Math. Phys. 16, 117 (1988)

12. Aldaya, V., de Azcarraga, J. A.: J. Math. Phys. 23, 1297 (1982)

13. Aldaya, V., de Azcarraga, J. A.: Proc. of the Conf. on "Constraint Theory and Relativistic Dynamics," Firence 1986. Longhi, G., Lusanna, L. (eds.): Singapore: World Scientific

14. Aldaya V., de Azcarraga, J. A.: Fortschr. Phys. 35 (6) 437 (1987)

15. Aldaya, V., de Azcarraga, J. A.: In: Modern developments in analytical mechanics. Benenti, S., Francaviglia, M., Lichnerovicz, A. (eds.): Acta Acad. Sci. Taurinensis (Torino) 117, [Suppl.] 1 (1983)

16. Souriau, J. M.: Structure des systemes dynamiques, Paris: Dunod 1970

17. Bargmann, V.: Ann. Math. 59, 1 (1954)

18. Balachandram, A. P., Gomm. H., Sorkin, R. D.: Nucl. Phys. B281, 573 (1987)

19. Isham, C. J., Linden, N.: Imperial College preprint TP/86-87/18

20. Itzykson, C., Zuber, J. B.: Quantum field theory. New York: McGraw Hill 1986

21. Scherk, J.: Rev. Mod. Phys. 47, 123 (1975)

22. Green, M. B., Schwarz, J. H., Witten, E.: Superstring Theory, vols. I and II. Cambridge: Cambridge University Press 1987

23. Kobayashi, S., Nomizu, K.: Foundations of differential geometry. N.Y.: Interscience vol. I, 1963

24. Becchi, C., Rouet, A., Stora, R.: Ann. Phys. 98, 287 (1976)

25. Bowick, M. J., Gursey, F.: Phys. Lett. B175, 182 (1986)

26. Thierry-Mieg, J.: J. Math Phys. 21, 2834 (1980)

27. Kostant, B.: Quantization and unitary representations. In: Lecture Notes in Mathematics, vol. 170. Berlin, Heidelberg, New York: Springer 1970

28. Simms, D. J., Woodhouse, N. M.: Lectures in geometric quantization. Berlin, Heidelberg, New York: Springer 1980; Sniatycki, J.: Geometric quantization and quantum mechanics, Berlin, Heidelberg, New York: Springer 1980; Woodhouse, N.M.: Geometric quantization. Oxford: Clarendon 1980 
29. Humphreys, J. E.: Introduction to Lie algebras and representation theory. Graduate texts in Mathematics vol. 9, Berlin, Heidelberg, New York: Springer 1972.

30. Bloore, F. J.: Lecture Notes in Mathematics, vol. 836, Berlin, Heidelberg, New York: Springer 1980

31. Greenberg, O.: Phys. Rev. Lett. 13, 598 (1964)

32. Witten, E.: Commun. Math. Phys. 114, 1 (1988)

33. Del Giudice, E., Di Vecchia, P., Fubini, S.: Ann. Phys. 70, 378 (1972)

34. Saclioglu, C.: CERN preprint TH.4854/87

Communicated by A. Jaffe

Received February 22, 1988 